\title{
High throughput approach to prepare high-density microcrystals in lipidic cubic phase for serial crystallography and fragment screening
}

\author{
Isabel Moraes ${ }^{1}$, Danny Axford², Agata Butryn², Pierre Aller², Tristan Kwan ${ }^{1}$, Peter J. Judge ${ }^{3}$. \\ ${ }^{1}$ National Physical Laboratory, Teddington, TW11 0LW, UK; \\ ${ }^{2}$ Diamond Light Source, Harwell Science and Innovation Campus, Didcot, OX11 ODE, UK; \\ ${ }^{3}$ Biochemistry Department, University of Oxford, South Parks Road, Oxford, OX1 3QU, UK \\ isabel.moraes@npl.co.uk
}

In recent years, serial crystallography has emerged as promising method for structural studies of integral membrane proteins. The possibility of collecting data from very small crystals at room temperature, with reduced radiation damage, has opened new opportunities to the membrane protein structural biology community. In particular in the field of time-resolved studies. However, one of the technical bottlenecks of the method is the production of large amounts of tiny optimized crystals in mesophases. Here, we present a simple and fast method to prepare hundreds of microliters of high-density microcrystals in lipidic cubic phase (LCP) for serial crystallography including time resolved measurements. This approach not only eliminates the need for large quantities of expensive gas-tight syringes, but also may be used as a high-throughput tool when screening conditions for the growth of high density well-diffracting crystals.

We also demonstrate, with practical examples, that this new approach is of great advantage to fragment drug discovery since it facilitates in situ crystal soaking with minimal disturbance to the crystals in LCP.

Finally, the method is economical and easily implemented in any standard crystallisation laboratory.

Keywords: Lipidic cubic phase, Membrane proteins, Serial Crystallography, Fragment screening 